\title{
Stability of Hybrid SDEs Driven by fBm
}

\author{
Wenyi Pei ${ }^{1,2,3 *}$ and Zhenzhong Zhang ${ }^{4}$ \\ ${ }^{1}$ School of Statistics and Mathematics, Zhejiang Gongshang University, Hangzhou, China, ${ }^{2}$ Collaborative Innovation Center of \\ Statistical Data Engineering, Technology and Application, Zhejiang Gongshang University, Hangzhou, China, ${ }^{3}$ College of \\ Information Science and Technology, Donghua University, Shanghai, China, ${ }^{4}$ Department of Statistics, Donghua University, \\ Shanghai, China
}

In this paper, the exponential stability of stochastic differential equations driven by multiplicative fractional Brownian motion (fBm) with Markovian switching is investigated. The quasi-linear cases with the Hurst parameter $H \in(1 / 2,1)$ and linear cases with $H \in(0,1 /$ 2) and $H \in(1 / 2,1)$ are all studied in this work. An example is presented as a demonstration.

Keywords: stochastic differential equation (SDEs), stability, fractional brownian motion, markovian switching, hybrid system

\section{INTRODUCTION}

In the natural world, it is a common phenomena that many practical systems may face random abrupt changes in their structures and parameters, such as environmental variance, changing of subsystem interconnections and so on. To deal with these abrupt changes, Markovian switching systems, a particular class of hybrid systems, are investigated and widely used $[1,2]$. Especially in signal processing, financial engineering, queueing networks, wireless communications and so on (see, e.g. [1, 3]).

In recent years, much attention has been paid to the stability of stochastic hybrid systems. For example, Mao [4] considers the exponential stability of general nonlinear stochastic hybrid systems. In [5], the criteria of moment exponential stability are obtained for stochastic hybrid delayed systems with Lévy noise in mean square. Zhou [6] investigates the pth moment exponential stability of the same systems. Some sufficient conditions for asymptotic stability in distribution of SDEs with Markovian switching are reported in [7]. See also [8,9] for more results about Markovian switching.

On the other hand, it is generally known that if $H \in(0,1 / 2)$ and $H \in(1 / 2,1),\left\{B_{t}^{H}\right\}_{t \geq 0}$ has a long range dependence, which means if we put

$$
r(n)=\operatorname{cov}\left(B_{1}^{H},\left(B_{n+1}^{H}-B_{n}^{H}\right)\right),
$$

then $\sum_{n=1}^{\infty} r(n)=\infty$. Besides, the process $\left\{B_{t}^{H}\right\}_{t \geq 0}$ is also self-similar for any $H \in(0,1)$. Since the pioneering work of Hurst [10,11] and Mandelbrot [12], the fractional Brownian motion has been suggested as a useful tool in many fields such as mathematical finance $[13,14]$ and weather derivatives [15]. Even though fractional Brownian motion is not a semimartingale, more and more financial models have been extended to $\mathrm{fBm}$ (see, e.g. $[16,17])$. Therefore, in this paper, the risk assets are described by hybrid stochastic systems driven by multiplicative $\mathrm{fBm}$. Then it is a natural and interesting question that under what conditions, this stochastic systems have some exponential stability. For the sake of clarity, we only consider the one dimensional cases. For more details about fractional noise, we refer the reader to [18-21].

The main purpose of this paper is to discuss the exponential stability of a risky asset, with price dynamics:

$$
\left\{\begin{array}{l}
\mathrm{d} X_{t}=f\left(X_{t}, t, r_{t}\right) \mathrm{d} t+g\left(X_{t}, t, r_{t}\right) \mathrm{d} B_{t}^{H}, \\
X_{0}=x_{0}>0
\end{array}\right.
$$

where $g\left(X_{t}, t, r_{t}\right)=\sigma\left(t, r_{t}\right) X_{t},\left\{r_{t}\right\}_{t \geq 0}$ is a Markov chain taking values in $\mathbb{S}=\{1,2, \ldots, N\},\left\{B_{t}^{H}\right\}_{t \geq 0}$ is a standard fractional Brownian motion. Moreover, $f\left(x, t, r_{t}\right): \mathbb{R} \times \mathbb{R}_{+} \times \mathbb{S} \rightarrow \mathbb{R}$ and $\sigma\left(t, r_{t}\right): \mathbb{R}_{+} \times \mathbb{S} \rightarrow \mathbb{R}$. 
In this paper, the initial value $x_{0}$ is assumed to be deterministic, otherwise more calculations about Wick product are required.

Equation 1 can be regarded as the result of the following $N$ fractional stochastic differential equations:

$$
\left\{\begin{array}{l}
\mathrm{d} X_{t}=f\left(X_{t}, t, i\right) \mathrm{d} t+g\left(X_{t}, t, i\right) \mathrm{d} B_{t}^{H}, \quad 1 \leq i \leq N, \\
X_{0}=x_{0}>0
\end{array}\right.
$$

switching from one to another according to the movement of $\left\{r_{t}\right\}_{t \geq 0}$.

Throughout this paper, unless otherwise specified, we let $C$ denote a general constant and $p$ denote a non-negative constant. Let $C^{2,1}\left(\mathbb{R} \times \mathbb{R}_{+} \times \mathbb{S} ; \mathbb{R}\right)$ denote the family of all real value functions on $\mathbb{R} \times \mathbb{R}_{+} \times \mathbb{S}$ which are continuously twice differentiable with respect to the first variables and once differentiable with respect to the second variables.

This paper is organized as follows. For the convenience of the reader, we briefly recall some of the basic results in Section 2. In Section 3, we investigate the solution and an extended Itô's Formula for the general hybrid fractional stochastic differential Equation 1. Section 3 is devoted to the linear cases. In this section the moment exponential stability and almost sure exponential stability are discussed respectively. In Section 4, some useful criteria for the exponential stability with respect to quasi-linear cases are presented. Finally, a numerical example and graphical illustration are presented in Section 6.

\section{PRELIMINARIES}

\subsection{Markov Chain}

Let $\left\{r_{t}\right\}_{t \geq 0}$ be a right-continuous Markov chain taking values in a finite state space $\mathbb{S}=\{1,2, \ldots, N\}$. The generator $Q=\left(q_{i j}\right)_{N \times N}$ is given by

$$
\mathbb{P}\left\{r_{t+\Delta}=j \mid r_{t}=i\right\}= \begin{cases}q_{i j} \triangle+o(\triangle), & \text { if } \quad i \neq j, \\ 1+q_{i j} \Delta+o(\triangle), & \text { if } i=j,\end{cases}
$$

where $\triangle>0$.

Here $q_{i j}$ is the transition rate from $i$ to $j$ if $i \neq j$. According to $[22,23]$, a continuous-time Markov chain $\left\{r_{t}\right\}_{t \geq 0}$ with generator $\mathrm{Q}=\left(q_{i j}\right)_{N \times N}$ can be represented as a stochastic integral with respect to a Poisson random measure. Then we have

$$
\mathrm{d} r_{t}=\int_{\mathbb{R}} h\left(r_{t-}, y\right) v(\mathrm{~d} t \times \mathrm{d} y)
$$

with initial condition $r_{0}=i_{0}$, where $\nu(d t \times d y)$ is a Poisson random measure with intensity $\mathrm{d} t \times m(\mathrm{~d} y)$. Here $m(\cdot)$ is the Lebesgue measure on $\mathbb{R}$.

Throughout this paper, unless otherwise specified, the Markov chain $\left\{r_{t}\right\}_{t \geq 0}$ has the invariant probability measure $\mu=\left(\mu_{i}\right)_{i \in \mathbb{S}}$ and is assumed to be independent of $\left\{B_{t}^{H}\right\}_{t \geq 0}$. Almost every sample path of the Markov chain $\left\{r_{t}\right\}_{t \geq 0}$ is assumed to be a rightcontinuous step function with a finite number of simple jumps in any finite time interval $[0, T]$. The generator $Q=\left(q_{i j}\right)_{N \times N}$ is assumed to be irreducible and conservative, i.e., $q_{i}:=-q_{i i}=$ $\sum_{i \neq j} q_{i j}<\infty$. For more details about Markovian switching we further refer the reader to [24-26].

\subsection{Fractional Brownian Motion and Wick Product}

We recall some of the basic results of $\mathrm{fBm}$ briefly, which will be needed throughout this paper. For more details about $\mathrm{fBm}$ we refer the reader to $[16,17,27,28]$. If $H \in(0,1 / 2) \cup(1 / 2,1)$, then the (standard) fractional Brownian motion with Hurst parameter $H$ is a continuous centered Gaussian process $\left\{B_{t}^{H}\right\}_{t \geq 0}$ with $\mathbb{E}\left(B_{t}^{H}\right)=0$ and covariance function:

$$
\mathbb{R}^{H}(s, t)=\mathbb{E}\left(B_{s}^{H} B_{t}^{H}\right)=\frac{1}{2}\left(|s|^{2 H}+|t|^{2 H}-|s-t|^{2 H}\right), \quad s, t \geq 0 .
$$

To simplify the representation, it is always assumed that $B_{0}^{H}=0$.

Besides, $\left\{B_{t}^{H}\right\}_{t \geq 0}$ has the following Wiener integral representation:

$$
B_{t}^{H}=\int_{0}^{t} K^{H}(t, s) \mathrm{d} W_{s},
$$

where $\left\{W_{t}\right\}_{t \geq 0}$ is a Wiener process and $K^{H}(t, s)$ is the kernel function defined by

$$
K_{H}(t, s)=c_{H} s^{\frac{1}{2}-H} \int_{0}^{t}(u-s)^{H-\frac{3}{2}} u^{H-\frac{1}{2}} \mathrm{~d} u,
$$

in which $c_{H}=\left(\frac{H(2 H-1)}{B\left(2-2 H, H-\frac{1}{2}\right)}\right)^{\frac{1}{2}}$, where $\mathrm{B}(\cdot, \cdot)$ is the Beta function, and $s<t$. In this paper, $\left\{B_{t}^{H}\right\}_{t \geq 0}$ generates a filtration $\left\{\mathcal{F}_{t}, t \geq 0\right\}$ with $\mathcal{F}_{t}=\sigma\left\{B_{s}^{H}, t \geq 0\right\}$. Denote $\left(\Omega, \mathcal{F}, P, \mathcal{F}_{t}\right)$ the complete probability space, with the filtration described above.

Let $\mathcal{I}$ be the set of all finite multi-indices $\alpha=\left(\alpha_{1}, \ldots, \alpha_{n}\right)$ for some $n \geq 1$ of non-negative integers. Denote $|\alpha|=\alpha_{1}+\cdots+\alpha_{n}$, and $\alpha !=\alpha_{1} ! \cdots \alpha_{n} !$

Define the Hermite polynomials:

$$
h_{n}(x)=(-1)^{n} e^{x^{2}} \frac{d^{n}}{d x^{n}}\left(e^{-x^{2}}\right), \quad n \geq 0,
$$

and Hermite functions:

$$
\tilde{h}_{n}(x)=\pi^{-\frac{1}{4}}(n !)^{-\frac{1}{2}} h_{n}(x) e^{-\frac{x^{2}}{4}}, \quad n \geq 0 .
$$

Let $S(\mathbb{R})$ denote the Schwartz space of rapidly decreasing infinitely differentiable $\mathbb{R}$-valued functions. Denote the dual space of $S(\mathbb{R})$ by $S^{\prime}(\mathbb{R})$. Define

$$
\mathcal{H}_{\alpha}(\omega)=\prod_{i=1}^{n} h_{\alpha_{i}}\left(\left\langle\tilde{h}_{i}(x), \omega\right\rangle\right),
$$

the product of Hermite polynomials. Consider a square integrable random variable

$$
F=F(\omega) \in L^{2}\left(S^{\prime}(\mathbb{R}), \mathcal{F}, P\right) .
$$

According to [17, 29], every $F(\omega)$ has a unique representation:

$$
F(\omega)=\sum_{\alpha \in \mathcal{I}} c_{\alpha} \mathcal{H}_{\alpha}(\omega)
$$

besides,

$$
\|F\|_{L^{2}(\omega)}^{2}=\sum_{\alpha \in \mathcal{I}} \alpha ! c_{\alpha}^{2}<\infty
$$


Definition 2.1. (Wick Product) For $F, G \in L^{2}\left(S^{\prime}(\mathbb{R}), \mathcal{F}, P\right)$, set $F(\omega)=\sum_{\alpha \in \mathcal{I}} \mathcal{c}_{\alpha} \mathcal{H}_{\alpha}(\omega)$ and $G(\omega)=\sum_{\beta \in \mathcal{I}} d_{\beta} \mathcal{H}_{\beta}(\omega)$. Their Wick product is defined by

$$
\begin{aligned}
F \diamond G(\omega) & =\sum_{\alpha, \beta \in \mathcal{I}} a_{\alpha} b_{\beta} \mathcal{H}_{\alpha+\beta}(\omega) \\
& =\sum_{\gamma \in \mathcal{I}}\left(\sum_{\alpha+\beta=\gamma} a_{\alpha} b_{\beta}\right) \mathcal{H}_{\gamma}(\omega) .
\end{aligned}
$$

\subsection{Malliavin Derivative}

Let $L^{p}:=L^{p}(\Omega, \mathcal{F}, P)$ be the space of all random variables $\Omega \rightarrow \mathbb{R}$, such that

$$
\|F\|_{p}=\mathbb{E}\left(|F|^{p}\right)^{1 / p}<\infty
$$

and let

$L_{\phi}^{2}\left(\mathbb{R}_{+}\right)=\left\{\left.f\left|f: \mathbb{R}_{+} \rightarrow \mathbb{R},\right| f\right|_{\phi} ^{2}:=\int_{0}^{\infty} \int_{0}^{\infty} f(s) f(t) \phi(s, t) \mathrm{d} s \mathrm{~d} t<\infty\right\}$,

where $\phi(s, t)=H(2 H-1)|s-t|^{2 H-2}$.

Definition 2.2. The $\phi$-derivative of $F \in L^{p}$ in the direction of $\Phi_{g}$ is defined by

$$
D_{\Phi_{g}} F(\omega)=\lim _{\delta \rightarrow 0} \frac{1}{\delta}\left\{F\left(\omega+\delta \int_{0}\left(\Phi_{g}\right)(u) \mathrm{d} u\right)-F(\omega)\right\},
$$

if the limit exists in $L^{p}$. Moreover if there exists a process $\left(D_{s}^{\phi} F_{s}, s \geq 0\right)$ such that

$$
D_{\Phi_{g}} F=\int_{0}^{\infty} D_{s}^{\phi} F_{s} g_{s} \mathrm{~d} s \quad \text { a.s. }
$$

for all $g \in L_{\phi}^{2}$, then $F$ is said to be $\phi$-differentiable.

According to $[16,30]$, let $\mathcal{A}(0, T)$ be the family of stochastic process on $[0, T]$ such that $F \in \mathcal{A}(0, T)$ if $\mathbb{E}|F|_{\phi}^{2}<\infty$ and $F$ is $\phi$-differentiable, the trace of $\left(D_{s}^{\phi} F_{t}, 0 \leq s \leq T, 0 \leq t \leq T\right)$ exists and $\mathbb{E} \int_{0}^{T}\left(D_{s}^{\phi} F_{s}\right)^{2} d s<\infty$, and for each sequence of partitions $\pi_{n}, n \in \mathbb{N}$ such that $\left|\pi_{n}\right| \rightarrow 0$, as $n \rightarrow \infty$. Moreover

$$
\sum_{i=0}^{n-1} \mathbb{E}\left\{\int_{t_{i}^{(n)}}^{t_{i+1}^{(n)}}\left|D_{s}^{\phi} F_{t_{i}^{(n)}}^{\pi}-D_{s}^{\phi} F_{s}\right| \mathrm{d} s\right\}^{2} \rightarrow 0,
$$

and

$$
\mathbb{E}\left|F^{\pi}-F\right|_{\phi}^{2} \rightarrow 0
$$

as $n \rightarrow \infty$. Here $\pi_{n}: 0=t_{0}^{(n)}<t_{1}^{(n)}<\ldots<t_{n}^{(n)}=T$, and $\left|\pi_{n}\right|=$ $\max _{i \in\{0,1, \ldots, n-1\}}\left\{t_{i+1}^{(n)}-t_{i}^{(n)}\right\}$.

Now we define the $B_{t}^{H}$-integral considered in [16].

Definition 2.3. Let $\left\{F_{t}\right\}_{t \geq 0}$ be a stochastic process such that $F \in \mathcal{A}(0, T)$. Define $\int_{0}^{T} F_{s} \mathrm{~d} B_{s}^{H}$ by

$$
\int_{0}^{T} F_{s} \mathrm{~d} B_{s}^{H}=\lim _{|\pi| \rightarrow 0} \sum_{i=0}^{n-1} F_{t_{i}}^{\pi} \diamond\left(B_{t_{i+1}}^{H}-B_{t_{i}}^{H}\right),
$$

where $|\pi|=\max _{i \in\{0,1, \ldots, n-1\}}\left\{t_{i+1}-t_{i}\right\}$.

Remark 2.1. : According to Theorem 3.6.1 in [16], if $F_{s} \in \mathcal{A}(0, T)$, then the stochastic integral satisfies $\mathbb{E} \int_{0}^{T} F_{s} \mathrm{~d} B_{s}^{H}=0$, and

$$
\mathbb{E}\left|\int_{0}^{T} F_{s} \mathrm{~d} B_{s}^{H}\right|^{2}=\mathbb{E}\left[\left(\int_{0}^{T} D_{s}^{\phi} F_{s} \mathrm{~d} s\right)^{2}+\left|1_{[0, T]} F\right|_{\phi}^{2}\right]
$$

What's more, according to Definition 3.4.1 in [16], the stochastic integral can be extended by

$$
\int_{\mathbb{R}} F_{t} \mathrm{~d} B_{t}^{H}:=\int_{\mathbb{R}} F_{t} \diamond W^{H}(t) d t
$$

where $F: \mathbb{R} \rightarrow(\mathcal{S})_{H}^{*}$ is a given function such that $F_{t} \diamond W^{H}(t)$ is $d t$ - integrable in $(\mathcal{S})_{H}^{*}$. Here $(\mathcal{S})_{H}^{*}$ is the fractional Hida distribution space defined by Definition 3.1.11 in [16]. In particular, the integral on $[0, \mathrm{~T}]$ can be defined by

$$
\int_{0}^{T} F_{t} \mathrm{~d} B_{t}^{H}=\int_{\mathbb{R}} F_{t} I_{[0, T]}(t) \mathrm{d} B_{t}^{H} .
$$

\section{HYBRID FRACTIONAL SYSTEMS}

In this section, firstly, we consider the existence and uniqueness of solution for Eq. 1. Then, an extended Itô's Formula is presented.

\subsection{Existence and Uniqueness}

To ensure the existence and uniqueness, we impose the following assumptions.

Assumption 3.1. Let $f=f(x, t, i): \mathbb{R} \times \mathbb{R}_{+} \times \mathbb{S} \rightarrow \mathbb{R}$ satisfy the hypothesises:

1) For each fixed $i \in \mathbb{S}, f(x, t, i)$ is measurable in all the arguments.

2) For each fixed $i \in \mathbb{S}$, there exists a constant $C>0$, such that

$|f(x, t, i)-f(y, t, i)| \leq C|x-y|, \quad \forall x, y \in \mathbb{R}, \quad \forall t \in \mathbb{R}_{+}$.

3) For each fixed $i \in \mathbb{S}$, there exists a constant $C>0$, such that

$$
|f(x, t, i)| \leq C(1+|x|), \quad \forall(x, t) \in \mathbb{R} \times \mathbb{R}_{+} .
$$

Assumption 3.2. Let $\sigma=\sigma(t, i): \mathbb{R}_{+} \times \mathbb{S} \rightarrow \mathbb{R}$ satisfy the hypothesises:

1) For each fixed $i \in \mathbb{S}, \sigma(t, i)$ is nonrandom;

2) For each fixed $i \in \mathbb{S}, \sigma(t, i) \in L^{\frac{1}{H}}\left(\mathbb{R}_{+}\right)$.

Lemma 3.1. : Let Assumptions 3.1, 3.2 hold. Then Eq. 1 has a unique solution.

Proof: The existence and uniqueness can be proved similar to that for Theorem 2.6 in [31], so we omit it here.

\subsection{The Itô Formula}

Next, we first review the results in $[16,30]$ on the Itô formula with respect to $\mathrm{fBm}$. Then we extend it to SDEs driven by $\mathrm{fBm}$ with Markovian switching.

Lemma 3.2. [16] (The Itô Formula) Let $\left(F_{u}, 0 \leq u \leq T\right)$ be a stochastic process in $\mathcal{A}(0, T)$. Assume that there exists an $\alpha>1-$ $H$ and $C>0$ such that 


$$
\mathbb{E}\left|F_{u}-F_{v}\right|^{2} \leq C|u-v|^{2 \alpha},
$$

where $|u-v| \leq \delta$ for some $\delta>0$ and

$$
\lim _{0 \leq u, v \leq t,|u-v| \rightarrow 0} \mathbb{E}\left|D_{u}^{\phi}\left(F_{u}-F_{v}\right)\right|^{2}=0 .
$$

Let $\sup _{0 \leq s \leq T}\left|G_{s}\right|<\infty$ and $\tilde{g}=\tilde{g}(x, t) \in C^{2,1}\left(\mathbb{R} \times \mathbb{R}_{+} ; \mathbb{R}\right)$ with bounded derivatives. Moreover, for $\eta_{t}=\int_{0}^{t} F_{u} \mathrm{~d} B_{u}^{H}$, it is assumed that $\mathbb{E} \int_{0}^{T}\left|F_{s} D_{s}^{\phi} \eta_{s}\right| \mathrm{d} s<\infty$ and $\left(\frac{\partial \tilde{g}}{\partial x}\left(s, \eta_{s}\right) F_{s}, s \in[0, T]\right)$ is in $\mathcal{A}(0, T)$. Denote $x_{t}=x_{0}+\int_{0}^{t} G_{u} \mathrm{~d} u+\int_{0}^{t} F_{u} \mathrm{~d} B_{u}^{H}, x_{0} \in \mathbb{R}$ for $t \in$ $[0, T]$. Let $\left(\frac{\partial \tilde{g}}{\partial x}\left(x_{s}, s\right) F_{s}, s \in[0, T]\right) \in \mathcal{A}(0, T), \mathbb{E}\left[\sup _{0 \leq s \leq t}\left|G_{s}\right|\right]<\infty$. Then for $t \in[0, T]$,

$$
\begin{aligned}
\tilde{g}\left(x_{t}, t\right) & =\tilde{g}\left(x_{0}, 0\right)+\int_{0}^{t} \frac{\partial \tilde{g}}{\partial s}\left(x_{s}, s\right) \mathrm{d} s+\int_{0}^{t} \frac{\partial \tilde{g}}{\partial x}\left(x_{s}, s\right) G_{s} \mathrm{~d} s \\
& +\int_{0}^{t} \frac{\partial \tilde{g}}{\partial x}\left(x_{s}, s\right) F_{s} \mathrm{~d} B_{s}^{H}+\int_{0}^{t} \frac{\partial^{2} \tilde{g}}{\partial x^{2}}\left(x_{s}, s\right) F_{s} D_{s}^{\phi} x_{s} \mathrm{~d} s .
\end{aligned}
$$

Here $D_{s}^{\phi} x_{s}$ is the Malliavin derivative defined in Definition 2.2. In particular, for the process
$X_{t}^{(i)}=X_{0}^{(i)}+\int_{0}^{t} f\left(X_{s}^{(i)}, s, i\right) \mathrm{d} s+\int_{0}^{t} g\left(X_{s}^{(i)}, s, i\right) \mathrm{d} B_{s}^{H}, \quad$ with each fixed $i \in \mathbb{S}$, we have that

$$
\begin{aligned}
F\left(X_{t}^{(i)}, t, i\right) & =F\left(X_{0}^{(i)}, 0, i\right)+\int_{0}^{t} \frac{\partial F}{\partial s}\left(X_{s}^{(i)}, s, i\right) \mathrm{d} s \\
& +\int_{0}^{t} \frac{\partial F}{\partial x}\left(X_{s}^{(i)}, s, i\right) f\left(X_{s}^{(i)}, s, i\right) \mathrm{d} s+\int_{0}^{t} \frac{\partial F}{\partial x}\left(X_{s}^{(i)}, s, i\right) g\left(X_{s}^{(i)}, s, i\right) \mathrm{d} B_{s}^{H} \\
& +\int_{0}^{t} \frac{\partial^{2} F}{\partial x^{2}}\left(X_{s}^{(i)}, s, i\right) g\left(X_{s}^{(i)}, s, i\right) D_{s}^{\phi} X_{s}^{(i)} \mathrm{d} s,
\end{aligned}
$$

Formally,

$$
\begin{array}{r}
\mathrm{d} F\left(X_{t}^{(i)}, t, i\right)=F_{t}\left(X_{t}^{(i)}, t, i\right) \mathrm{d} t+F_{x x}\left(X_{t}^{(i)}, t, i\right) g\left(X_{t}^{(i)}, t, i\right) D_{s}^{\phi} X_{s}^{(i)} \mathrm{d} t \\
+F_{x}\left(X_{t}^{(i)}, t, i\right) f\left(X_{t}^{(i)}, t, i\right) \mathrm{d} t+F_{x}\left(X_{t}^{(i)}, t, i\right) g\left(X_{t}^{(i)}, t, i\right) \mathrm{d} B_{t}^{H},
\end{array}
$$

Let

$$
\begin{aligned}
\mathcal{L}^{(i)} F\left(X_{t}^{(i)}, t, i\right) & =F_{t}\left(X_{t}^{(i)}, t, i\right)+F_{x}\left(X_{t}^{(i)}, t, i\right) f\left(X_{t}^{(i)}, t, i\right) \\
& +F_{x x}\left(X_{t}^{(i)}, t, i\right) g\left(X_{t}^{(i)}, t, i\right) D_{s}^{\phi} X_{s} .
\end{aligned}
$$

Substituting Eq. 3 into Eq. 2, we get

$$
\begin{aligned}
F\left(X_{t}^{(i)}, t, i\right) & =F\left(X_{0}^{(i)}, 0, i\right)+\int_{0}^{t} \mathcal{L}^{(i)} F\left(X_{s}^{(i)}, s, i\right) \mathrm{d} s \\
& +\int_{0}^{t} F_{x}\left(X_{s}^{(i)}, s, i\right) g\left(X_{s}^{(i)}, s, i\right) \mathrm{d} B_{s}^{H} .
\end{aligned}
$$

In the sequel of this paper, unless otherwise specified, we let the coefficients of Eq. 1 satisfy the conditions in Lemma 3.2, for each fixed $i \in \mathbb{S}$. Set $V\left(X_{t}, t, r_{t}\right) \in C^{2,1}\left(\mathbb{R} \times \mathbb{R}_{+} \times \mathbb{S} ; \mathbb{R}_{+}\right)$. Next we consider the Itô formula which reveals how $V$ maps $\left(X_{t}, t, r_{t}\right)$ into a new process $V\left(X_{t}, t, r_{t}\right)$, where $\left\{X_{t}\right\}_{t \geq 0}$ is a stochastic process with the stochastic differential Eq. 1 .

Lemma 3.3. If $V\left(X_{t}, t, r_{t}\right) \in C^{2,1}\left(\mathbb{R} \times \mathbb{R}_{+} \times \mathbb{S} ; \mathbb{R}_{+}\right)$, then for any 0 $\leq s<t$,

$$
\begin{aligned}
\mathbb{E} V\left(X_{t}, t, r_{t}\right) & =\mathbb{E} V\left(X_{s}, s, r_{s}\right)+\mathbb{E} \int_{s}^{t} \mathcal{A} V\left(X_{u}, u, r_{u}\right) \mathrm{d} u \\
& +\mathbb{E} \int_{s}^{t} V_{x}\left(X_{u}, u, r_{u}\right) g\left(X_{u}, u, r_{u}\right) \mathrm{d} B_{u}^{H}
\end{aligned}
$$

where $\mathcal{A} V$ is defined by

$$
\mathcal{A} V(x, t, i)=\mathcal{L}^{(i)} V(x, t, i)+\sum_{j=1}^{N} \gamma_{i j} V(x, t, j) .
$$

Proof: This result can be obtained similarly to that in [31] and we therefore omit it. For further details we also refer to [2, 23].

\section{LINEAR HYBRID FRACTIONAL SYSTEMS}

There are many models for financial markets with $\mathrm{fBm}$ (see, e.g. [16]). The simplest nontrivial type of market is the $\mathrm{fBm}$ version of the classical Black Scholes market, in which linear fractional SDEs is used. Thus, we would like to give some new criteria for switching linear fractional SDEs with $H \in\left(0, \frac{1}{2}\right)$ or $H \in\left(\frac{1}{2}, 1\right)$. At first, we present a definition and a useful lemma.

Definition 4.1. Let $H \in(0,1)$. The operator $M$ is defined on functions $f \in S(\mathbb{R})$ by

$$
M f(x)=-\frac{d}{d x} \frac{C_{H}}{(H-1 / 2)} \int_{\mathbb{R}}(t-s)|t-x|^{H-\frac{3}{2}} f(t) \mathrm{d} t
$$

where

$$
\begin{aligned}
& C_{H}=\left\{2 \Gamma\left(H-\frac{1}{2}\right) \cos \left[\frac{\pi}{2}\left(H-\frac{1}{2}\right)\right]\right\}^{-1} \\
& {[\Gamma(2 H+1) \sin (\pi H)]^{\frac{1}{2}} .}
\end{aligned}
$$

Here $\Gamma(\cdot)$ denotes the classical Gamma function.

According to [16], Eq. 6 can be restated as follows.

For $H \in(0,1 / 2)$, we have

$$
M f(x)=C_{H} \int_{\mathbb{R}} \frac{f(x-t)-f(x)}{|t|^{3 / 2-H}} \mathrm{~d} t .
$$

For $H=1 / 2$, we have

$$
M f(x)=f(x) .
$$

For $H \in(1 / 2,1)$, we have

$$
M f(x)=C_{H} \int_{\mathbb{R}} \frac{f(t)}{|t-x|^{3 / 2-H}} \mathrm{~d} t .
$$

Lemma 4.1. Let $\left\{r_{t}\right\}_{t \geq 0}$ be a right-continuous Markov chain which takes values in a finite state space $\mathbb{S}=\{1,2, \ldots, N\}$. Assume that it is irreducible and positive recurrent with invarient measure $\mu$. If $\alpha(\cdot): \mathbb{S} \rightarrow \mathbb{R}$ is a function verifying

$$
\alpha:=\sum_{i \in \mathbb{S}} \mu(i) \alpha(i)>0 .
$$

Then there exists constants $C, c>0$ such that:

$$
c e^{-\alpha t} \leq \mathbb{E}\left[e^{-\int_{0}^{t} \alpha\left(r_{s}\right) \mathrm{d} s}\right] \leq C e^{-\alpha t},
$$

for any initial condition $r_{0}$ and every $t \geq 0$. 
Proof: It is a consequence of Perron-Frobenius theorem and the study of eigenvalues. See Proposition 4.1 in [25], Proposition 4.2 in [25], and Lemma 2.7 in [26], for further details.

In Eq. 1, let us consider the case $g\left(x, t, r_{t}\right)=\sigma\left(t, r_{t}\right) x=t^{h} b\left(r_{t}\right) x$, $f\left(x, t, r_{t}\right)=\alpha\left(r_{t}\right) x$, where $\alpha(i)$ and $b(i)$ are constants for each $i \in \mathbb{S}$. This means that we are considering the following linear equation:

$$
\left\{\begin{aligned}
\mathrm{d} X_{t} & =\alpha\left(r_{t}\right) X_{t} \mathrm{~d} t+\sigma\left(t, r_{t}\right) X_{t} \mathrm{~d} B_{t}^{H}, \\
X_{0} & =x_{0} .
\end{aligned}\right.
$$

Set $\bar{b}=\max \{|b(i)|, i \in \mathbb{S}\}$ and $\underline{b}=\min \{|b(i)|, i \in \mathbb{S}\} . x_{0}$ is the deterministic initial value. For the sake of clarity, we firstly set $h=$ $1 / 2-H$.

\section{1 pth Moment Exponential Stability}

Theorem 4.1. Let $\left\{X_{t}\right\}_{t \geq 0}$ be the solution of Eq. 7 with $H \in(1 / 2$, 1), $h=1 / 2-H$.

1) If $\sum_{i \in S} \mu_{i} \alpha(i)-\frac{(1-p) \underline{b}^{2}}{2}<0$, then $\lim \sup \frac{1}{t} \log \left(\mathbb{E}\left|X_{t}\right|^{p}\right)<0$.

2) If $\sum_{i \in \mathbb{S}} \mu_{i} \alpha(i)-\frac{(1-p) \bar{b}^{2}}{2}>0$, then $\lim _{t \rightarrow \infty}^{t \rightarrow \infty} \mathbb{E}\left|X_{t}\right|^{p}=\infty$.

Proof. According to [16], without too many calculations, we obtain that $\left\{X_{t}\right\}_{t \geq 0}$ has the following form:

$$
X_{t}=x_{0} \exp \left[\int_{0}^{t} \sigma\left(r_{s}\right) \mathrm{d} B_{s}^{H}+\int_{0}^{t} \alpha\left(r_{s}\right) \mathrm{d} s-\frac{1}{2} \int_{\mathbb{R}}\left(M_{s}\left(\sigma\left(t, r_{s}\right) I_{[0, t]}(s)\right)\right)^{2} \mathrm{~d} s\right],
$$

where $M_{s}$ is the operator $M$ acting on the variable $s$. Let $x_{0} \neq 0$. It follows from Eq. 8 that

$$
\begin{aligned}
& \mathbb{E}\left|X_{t}\right|^{p} \\
= & \mathbb{E}\left(\left|x_{0}\right| \exp \left[\int_{0}^{t} \sigma\left(t, r_{s}\right) \mathrm{d} B_{s}^{H}+\int_{0}^{t} \alpha\left(r_{s}\right) \mathrm{d} s-\frac{1}{2} \int_{\mathbb{R}}\left(M_{s}\left(\sigma\left(t, r_{s}\right) I_{[0, t]}(s)\right)\right)^{2} \mathrm{~d} s\right]\right)^{p}
\end{aligned}
$$

We then see from Eq. 9 that

$$
\mathbb{E}\left|X_{t}\right|^{p}=\mathbb{E}\left(\exp \left(p\left[\int_{0}^{t} \alpha\left(r_{s}\right) \mathrm{d} s-\frac{1-p}{2} \int_{\mathbb{R}}\left(M_{s}\left(\sigma\left(t, r_{s}\right) I_{[0, t]}(s)\right)\right)^{2} \mathrm{~d} s\right]\right) \zeta_{t}\right),
$$

where

$$
\begin{aligned}
& \zeta_{t}=\left|x_{0}\right|^{p} \exp \int_{0}^{t} p \sigma\left(s, r_{s}\right) \mathrm{d} B_{s}^{H}-\frac{p^{2}}{2} \\
& \int_{\mathbb{R}}\left(M_{s}\left(\sigma\left(t, r_{s}\right) I_{[0, t]}(s)\right)\right)^{2} \mathrm{~d} s .
\end{aligned}
$$

Noting that $\zeta_{t}$ is the solution to the equation

$$
\mathrm{d} \zeta_{t}=p \sigma\left(t, r_{t}\right) \zeta_{t} \mathrm{~d} B_{t}^{H},
$$

with initial value $\zeta_{0}=\left|x_{0}\right|^{p}$. Thus

$$
\zeta_{t}=\left|x_{0}\right|^{p}+\int_{0}^{t} p \sigma\left(t, r_{s}\right) \mathrm{d} B_{s}^{H},
$$

which yields

$$
\mathbb{E} \zeta_{t}=\mathbb{E}\left[\left|x_{0}\right|^{p}+\int_{0}^{t} p \sigma\left(t, r_{s}\right) \mathrm{d} B_{s}^{H}\right]=\left|x_{0}\right|^{p} .
$$

Substituting Eq. 11 into Eq. 10 gives

$\mathbb{E}\left|X_{t}\right|^{p}=\mathbb{E} \exp \left(p\left[\int_{0}^{t} \alpha\left(r_{s}\right) \mathrm{d} s-\frac{1-p}{2} \int_{\mathbb{R}}\left(M_{s}\left(\sigma\left(r_{s}\right) I_{[0, t]}(s)\right)\right)^{2} \mathrm{~d} s\right]\right)\left|x_{0}\right|^{p}$.

Note that

$$
\begin{aligned}
& \int_{\mathbb{R}}\left(M_{s}\left(\underline{b} s^{h} I_{[0, t]}(s)\right)\right)^{2} \mathrm{~d} s \leq \int_{\mathbb{R}}\left(M_{s}\left(\sigma\left(t, r_{s}\right) I_{[0, t]}(s)\right)\right)^{2} \\
& \mathrm{~d} s \leq \int_{\mathbb{R}}\left(M_{s}\left(\bar{b} s^{h} I_{[0, t]}(s)\right)\right)^{2} \mathrm{~d} s .
\end{aligned}
$$

Consequently, by Definition 4.1 and [16], one has

$$
\underline{b}^{2} t \leq \int_{\mathbb{R}}\left(M_{s}\left(\sigma\left(r_{s}\right) I_{[0, t]}(s)\right)\right)^{2} \mathrm{~d} s \leq \bar{b}^{2} t .
$$

Making use of Eqs 12, 13, we obtain that

$$
\begin{aligned}
& \mathbb{E} \exp \left(p\left[\int_{0}^{t} \alpha\left(r_{s}\right) \mathrm{d} s-\frac{1-p^{2}}{2} \bar{b}^{2} t\right)\right]\left|x_{0}\right|^{p} \leq \mathbb{E}\left|X_{t}\right|^{p} \\
& \leq \mathbb{E} \exp \left(p\left[\int_{0}^{t} \alpha\left(r_{s}\right) \mathrm{d} s-\frac{1-p}{2} \underline{b}^{2} t\right)\right]\left|x_{0}\right|^{p} .
\end{aligned}
$$

Therefore, by Lemma 4.1 and Eq. 12, the required assertions follow. The proof is complete.

Theorem 4.2. Let $\left\{X_{t}\right\}_{t \geq 0}$ be the solution of Eq. 7 with $H \in(0,1 /$ 2), $h=1 / 2-H$.

1) If $\sum_{i \in S} \mu_{i} \alpha(i)<\frac{(1-p) \underline{b}^{2}}{2}$, then $\limsup _{t \rightarrow \infty} \frac{1}{t} \log \left(\mathbb{E}\left|X_{t}\right|^{p}\right)<0$.

2) If $\sum_{i \in \mathbb{S}} \mu_{i} \alpha(i)>\frac{(1-p) \bar{b}^{2}}{2}$, then $\lim _{t \rightarrow \infty}^{t \rightarrow \infty} \mathbb{E}\left|X_{t}\right|^{p}=\infty$.

Proof: Similar to Theorem 4.1, we write the solution as follows.

$\mathbb{E}\left|X_{t}\right|^{p}=\mathbb{E} \exp \left(p\left[\int_{0}^{t} \alpha\left(r_{s}\right) \mathrm{d} s+\frac{p-1}{2} \int_{\mathbb{R}}\left(M_{s}\left(\sigma\left(r_{s}\right) I_{[0, t]}(s)\right)\right)^{2} \mathrm{~d} s\right]\right)\left|x_{0}\right|^{p}$.

Note that $M_{s}$ is the operator $M$ acting on the variable $s$, where

$$
M f(x)=C_{H} \int_{\mathbb{R}} \frac{f(x-t)-f(x)}{|t|^{3 / 2-H}} \mathrm{~d} t .
$$

According to [16], we also have that

$$
\underline{b}^{2} t \leq \int_{\mathbb{R}}\left(M_{s}\left(\sigma\left(t, r_{s}\right) I_{[0, t]}(s)\right)\right)^{2} \mathrm{~d} s \leq \bar{b}^{2} t
$$

Consequently, by Lemma 4.1, the result follows. The proof is complete.

Remark 4.1. In the above Theorems 4.1, 4.2, the parameter $h$ is supposed to be $H-1 / 2$. Noting that by Eqs 13, 15 and together with the Definition 4.1, the stability of solution for 
Eq. 7 with $h<1 / 2-H$ or $h>1 / 2-H$ can be deduced respectively without too many difficulties.

Remark 4.2. Take $H=1 / 2$. It's easy to show that if $\sum_{i \in S} \mu_{i} \alpha(i)=\alpha<\frac{(1-p) \underline{\sigma}^{2}}{2}$, then $\lim \sup \frac{1}{t} \log \left(\mathbb{E}\left|X_{t}\right|^{p}\right)<0$, and if $\sum_{i \in \mathbb{S}} \mu_{i} \alpha(i)=\alpha>\frac{(1-p) \bar{\sigma}^{2}}{2}$, then $\lim _{t \rightarrow \infty} \mathbb{E}\left|X_{t}\right|^{p}=\infty$, which coincide with the results of SDEs driven by Brownian motion in $[4,32]$.

\subsection{Almost Sure Exponential Stability}

To proceed, we need to introduce the definition of almost sure stability and a useful lemma.

Definition 4.2. The equilibrium point $x=0$ is said to be almost surely exponential stable if

$$
\limsup _{t \rightarrow \infty} \frac{1}{t} \log \left|X_{t}\right|<0 \quad \text { a.s. }
$$

for any $x_{0} \in \mathbb{R}$.

Lemma 4.2. (Law of the iterated logarithm) For a standard $\mathrm{fBm}$ $\left\{B_{t}^{H}\right\}_{t \geq 0}$, we have that

$$
\limsup _{t \rightarrow \infty} \frac{B_{t}^{H}}{t^{H} \sqrt{\log \log t}}=C_{H},
$$

where $C_{H}>0$ is a suitable constant.

Proof: By [33], we have

$$
\limsup _{t \rightarrow 0^{+}} \frac{B_{t}^{H}}{t^{H} \sqrt{\log \log t^{-1}}}=c_{H},
$$

where $c_{H}$ is a suitable constant. Then the thesis follows by the selfsimilarity of $\mathrm{fBm}$ and a change of variable $t \rightarrow 1 / t$.

For the sake of clarity, we firstly set $h=0$. Namely, let us consider

$$
\left\{\begin{array}{l}
d X_{t}=\alpha\left(r_{t}\right) X_{t} \mathrm{~d} t+b\left(r_{t}\right) X_{t} \mathrm{~d} B_{t}^{H}, \\
X_{0}=x_{0} .
\end{array}\right.
$$

Noting that Eq. 17 is exactly the geometry $\mathrm{fBm}$ with Markovian Switching. We proceed to discuss the almost sure exponential stability about it.

Theorem 4.3. 1) If $0<H<1 / 2$, the equilibrium point $\mathrm{x}=0$ of the system Eq. 17 is almost surely exponential stable when $\sum_{i \in \mathbb{S}} \mu_{i} \alpha(i)<0$, but unstable when $\left.\sum_{i \in \mathbb{S}} \mu_{i} \alpha(i)>0 ; 2\right)$ If $H=1 / 2$, the equilibrium point $x=0$ of the system Eq. 17 is almost surely exponential stable when $\sum_{i \in \mathbb{S}} \mu_{i} \alpha(i)<\frac{1}{2} \underline{b}^{2}$, but unstable when $\left.\sum_{i \in \mathbb{S}} \mu_{i} \alpha(i)>\frac{1}{2} \bar{b}^{2} ; 3\right)$ If $1 / 2<H<1$, the equilibrium point $x=$ 0 of the system Eq. 17 is almost surely exponential stable for all parameters $\alpha(i)$ and $\sigma(i), i \in \mathbb{S}$.

Proof: Define

$$
\lambda=\limsup _{t \rightarrow \infty} \frac{1}{t} \log \left|X_{t}\right|
$$

From Eqs 8, 16, we have

$$
\begin{aligned}
& \lambda=\limsup _{t \rightarrow \infty} \frac{1}{t} \log \left|X_{t}\right| \\
& =\limsup _{t \rightarrow \infty} \frac{1}{t} \log \left|x_{0} \exp \left[\int_{0}^{t} \sigma\left(r_{s}\right) \mathrm{d} B_{s}^{H}+\int_{0}^{t} \alpha\left(r_{s}\right) \mathrm{d} s-\frac{1}{2} \int_{\mathbb{R}}\left(M_{s}\left(\sigma\left(r_{s}\right) I_{[0, t]}(s)\right)\right)^{2} \mathrm{~d} s\right]\right| \\
& =\lim _{t \rightarrow \infty}\left(\sum_{i \in \mathbb{S}} \mu_{i} \alpha(i)-\frac{1}{2 t} \int_{\mathbb{R}}\left(M_{s}\left(\sigma\left(r_{s}\right) I_{[0, t]}(s)\right)\right)^{2} \mathrm{~d} s\right) .
\end{aligned}
$$

By Definition 4.1 and [16], one has

$$
\underline{b}^{2} t^{2 H} \leq \int_{\mathbb{R}}\left(M_{s}\left(b\left(r_{s}\right) I_{[0, t]}(s)\right)\right)^{2} \mathrm{~d} s \leq \bar{b}^{2} t^{2 H} .
$$

Making use of Eq. 18, we get

$$
\lambda=\left\{\begin{array}{lr}
\sum_{i \in \mathbb{S}} \mu_{i} \alpha(i), & 0<H<1 / 2 ; \\
-\infty, & 1 / 2<H<1 .
\end{array}\right.
$$

Especially, when $H=1 / 2$, we have that

$$
\sum_{i \in \mathbb{S}} \mu_{i} \alpha(i)-\frac{1}{2} \bar{b}^{2} \leq \lambda \leq \sum_{i \in \mathbb{S}} \mu_{i} \alpha(i)-\frac{1}{2} \underline{b}^{2} .
$$

Therefore, the required results follows. The proof is complete.

Remark 4.3. Making use of Eq. 18, one can discuss the almost sure exponential stability for Eq. 7 with $h \neq 0$. The proofs are similar to Theorem 4.3 and are omitted.

\section{QUASI-LINEAR HYBRID FRACTIONAL SYSTEMS}

We now apply the extended Itô Formula in Section 3 to discuss the stability for quasi-linear fractional SDEs with Markovian switching.

Theorem 5.1. : Let Assumptions 3.1, 3.2 hold. If there exists a function $V \in C^{2,1}\left(\mathbb{R} \times \mathbb{R}_{+} \times \mathbb{S} ; \mathbb{R}_{+}\right)$and positive constants $a_{1}, a_{2}$, $b$ and $p \geq 1$, such that

$$
\begin{gathered}
a_{1}\left|X_{t}\right|^{p} \leq\left|V\left(X_{t}, t, i\right)\right| \leq a_{2}\left|X_{t}\right|^{p}, \\
\mathcal{L}^{(i)} V\left(X_{t}, t, i\right) \leq-b\left|X_{t}\right|^{p},
\end{gathered}
$$

for all $X_{t} \in \mathbb{R}, t \geq t_{0}, i \in \mathbb{S}$.

Then the solution of Eq. 1 is $p$ th moment exponential stable. More precisely,

$$
\limsup _{t \rightarrow \infty} \frac{1}{t} \log \left(\mathbb{E}\left|X_{t}\right|^{p}\right)<0
$$

Proof: According to Lemma 3.1, Eq. 1 has a unique solution. Denote it $\left\{X_{t}\right\}_{t \geq 0}$. Set

$$
U\left(X_{t}, t, i\right)=e^{\lambda t} V\left(X_{t}, t, i\right),
$$

where $\lambda \in\left(\eta, \frac{b}{a_{2}}\right), \eta>0$. Making use of Definition 2.3 and Lemma 3.2, one has $\mathcal{A} U=e^{\lambda t}(\lambda V+\mathcal{A} V)$ and $\left(U_{x} g, s \in[0, T]\right) \in \mathcal{A}(0, T)$.

Applying the conditions Eq. 19, 20, together with the generalized ItôEq. 5 and Remark 2.1, we obtain that for any $t \in[0, T]$ 


$$
\begin{aligned}
a_{1} e^{\eta t} \mathbb{E}\left|X_{t}\right|^{p} \leq & \mathbb{E} U\left(X_{t}, t, i\right)=\mathbb{E} V\left(X_{0}, 0, r_{0}\right)+\mathbb{E} \int_{0}^{t} \mathcal{A} U \mathrm{~d} s \\
& \quad+\mathbb{E} \int_{0}^{t} U_{x} g \mathrm{~B}^{H}=\mathbb{E} V\left(X_{0}, 0, r_{0}\right)+\mathbb{E} \int_{0}^{t} \mathcal{L}^{\left(r_{s}\right)} U \mathrm{~d} s \\
= & \mathbb{E} V\left(X_{0}, 0, r_{0}\right)+\mathbb{E} \int_{0}^{t} e^{\lambda s}(\lambda V+\mathcal{A} V) \mathrm{d} s \leq \mathbb{E} V\left(X_{0}, 0, r_{0}\right) \\
& +\mathbb{E} \int_{0}^{t} e^{\lambda s}\left(\lambda a_{2}-b\right)\left|X_{t}\right|^{p} \mathrm{~d} s .
\end{aligned}
$$

Thus we obtain that

$$
a_{1} e^{\eta t} \mathbb{E}\left|X_{t}\right|^{p} \leq \mathbb{E} V\left(X_{0}, 0, r_{0}\right)+\mathbb{E} \int_{0}^{t} e^{\lambda s}\left(\lambda a_{2}-b\right)\left|X_{t}\right|^{p} \mathrm{~d} s .
$$

Dividing both sides of Eq. 21 by $a_{1} e^{\eta t}$, noting that $\lambda a_{2}-b<0$, we get

$$
\begin{aligned}
\mathbb{E}\left|X_{t}\right|^{p} & \leq \frac{e^{-\eta t}}{a_{1}} \mathbb{E} V\left(X_{0}, 0, r_{0}\right)+\frac{e^{-\eta t}}{a_{1}} \mathbb{E} \int_{0}^{t} e^{\lambda s}\left(\lambda a_{2}-b\right)\left|X_{t}\right|^{p} \mathrm{~d} s \\
& \leq \frac{e^{-\eta t}}{a_{1}} \mathbb{E} V\left(X_{0}, 0, r_{0}\right) .
\end{aligned}
$$

Consequently,

$$
\sup _{t \in[0, T]} a_{1} e^{\eta t} \mathbb{E}\left|X_{t}\right|^{p} \leq \mathbb{E} V\left(X_{0}, 0, r_{0}\right) .
$$

Letting $T \rightarrow \infty$ gives

$$
\sup _{t \geq 0} \mathbb{E}\left|X_{t}\right|^{p} \leq \frac{e^{-\eta t}}{a_{1}} \mathbb{E} V\left(X_{0}, 0, r_{0}\right),
$$

and the required assertion follows. The proof is complete.

In the sequel of this section, we give another useful criterion and prove it briefly.

Theorem 5.2. Assume that Eq. 1 has a unique solution and there exist a function $V \in C^{2,1}\left(\mathbb{R} \times \mathbb{R}_{+} \times \mathbb{S} ; \mathbb{R}_{+}\right)$and positive constants $b_{1}, b_{2}, p \geq 1$ and $\beta_{i} \in \mathbb{R}$ such that for all $x \in \mathbb{R}, t \geq t_{0}, i \in \mathbb{S}$,

$$
\begin{gathered}
b_{1}|x|^{p} \leq|V(x, t, i)| \leq b_{2}|x|^{p}, \\
\mathcal{L}^{(i)} V(x, t, i) \leq \beta_{i} V(x, t, i),
\end{gathered}
$$

and

$$
\sum_{i \in \mathbb{S}} \mu_{i} \beta_{i}<0
$$

Then Eq. 1 is $p$ th moment exponential stable.

Proof: Set $\bar{\beta}_{i}=\frac{1}{\theta} \beta_{i}$, where $\theta \in(0,1)$. Let $\delta=-\sum_{i \in \mathbb{S}} \mu_{i} \bar{\beta}_{i}=-\mu \bar{\beta}$. Let 1 denote the vector which all elements are 1 . Then,

$$
\mu(\bar{\beta}+\delta 1)=\mu \bar{\beta}+\delta=-\delta+\delta=0 .
$$

By [1], Eq. 22 implies the Poisson equation:

$$
Q c=\bar{\beta}+\delta 1 \text {. }
$$

Note that Eq. 23 has the solution $c=\left(c_{1}, \ldots, c_{N}\right)^{T}$. Hence,

$$
-\delta=\bar{\beta}_{i}-\sum_{j=1}^{N} q_{i j} c_{j}, \quad i \in \mathbb{S} .
$$

For each $i \in \mathbb{S}$, set $U(x, t, i)=\left(1-\theta c_{i}\right) V(x, t, i)$, where $\theta \in(0,1)$ is already defined and sufficiently small satisfying $1-\theta c_{i}>0$.

Then, for any $t \in[0, T]$ we get

$$
\begin{aligned}
\mathcal{A} U(x, t, i) & =\left(1-\theta c_{i}\right) \mathcal{L}^{(i)} V(x, t, i)+\sum_{i \neq j} q_{i j}(U(x, t, j)-U(x, t, i)) \\
& =\left(1-\theta c_{i}\right) \mathcal{L}^{(i)} V(x, t, i)-\theta V(x, t, i) \sum_{i \neq j} q_{i j}\left(c_{j}-c_{i}\right) \\
& \leq\left(1-\theta c_{i}\right) \theta V(x, t, i)\left[\bar{\beta}_{i}-\sum_{i \neq j} q_{i j} \frac{c_{j}-c_{i}}{\left(1-\theta c_{i}\right)}\right] .
\end{aligned}
$$

According to $[1,31]$, one has

$$
\begin{aligned}
& \sum_{i \neq j} q_{i j} \frac{c_{j}-c_{i}}{\left(1-\theta c_{i}\right)}=\sum_{i \neq j} q_{i j} c_{j}+\sum_{i \neq j} q_{i j} \frac{\theta c_{i} c_{j}-c_{i}}{1-\theta c_{i}} \\
= & \sum_{j=1}^{N} q_{i j} c_{j}+\sum_{i \neq j} q_{i j} \frac{c_{i}\left(c_{j}-c_{i}\right)}{1-\theta c_{i}} \theta=\sum_{j=1}^{N} q_{i j} c_{j}+o(\theta) .
\end{aligned}
$$

Making use of Eqs 25, 26, we obtain that

$$
\mathcal{A} U(x, t, i) \leq\left(1-\theta c_{i}\right) \theta V(x, t, i)\left[\bar{\beta}_{i}-\sum_{j=1}^{N} q_{i j} c_{j}+o(\theta)\right] .
$$

Substituting Eq. 24 into Eq. 27, we get

$$
\mathcal{A} U(x, t, i) \leq\left(1-\theta c_{i}\right) \theta V(x, t, i)[o(\theta)-\delta]=\kappa U(x, t, i),
$$

where $\kappa<0$. Making use of Theorem 5.1, the desired criterion follows.

On the other hand, we can prove it in another way. Set $\eta>0$ and $\lambda \in(\eta,-\kappa)$. Define

$$
\bar{U}\left(X_{t}, t, i\right)=\frac{e^{\lambda t}}{1-\theta c_{i}} U\left(X_{t}, t, i\right)
$$

Compute

$$
\begin{aligned}
& b_{1} e^{\eta t} \mathbb{E}\left|X_{t}\right|^{p} \leq \mathbb{E} \bar{U}\left(X_{t}, t, i\right)=\mathbb{E} U\left(X_{0}, 0, i_{0}\right)+\mathbb{E} \int_{0}^{t} \mathcal{A} \bar{U} \mathrm{~d} s+\mathbb{E} \int_{0}^{t} \bar{U}_{x} g \mathrm{~d} B_{s}^{H} \\
& =\mathbb{E} U\left(X_{0}, 0, i_{0}\right)+\mathbb{E} \int_{0}^{t} e^{\lambda s}(\lambda U+\mathcal{A} U) \mathrm{d} s \leq \mathbb{E} U\left(X_{0}, 0, i_{0}\right)+\mathbb{E} \int_{0}^{t} e^{\lambda s}(\lambda+\kappa) U \mathrm{~d} s \\
& =\mathbb{E} V\left(x_{0}, 0, i_{0}\right)+\mathbb{E} \int_{0}^{t} e^{\lambda s}(\lambda+\kappa) V \mathrm{~d} s \\
& \leq \mathbb{E} V\left(x_{0}, 0, i_{0}\right)+\mathbb{E} \int_{0}^{t} e^{\lambda s}(\lambda+\kappa) b_{2}\left|X_{t}\right|^{p} \mathrm{~d} s .
\end{aligned}
$$

Thus we obtain that

$$
b_{1} e^{\eta t} \mathbb{E}\left|X_{t}\right|^{p} \leq V\left(x_{0}, 0, i_{0}\right)+\mathbb{E} \int_{0}^{t} e^{\lambda s} b_{2}(\lambda+\kappa)\left|X_{t}\right|^{p} \mathrm{~d} s,
$$

Dividing both sides of Eq. 28 by $b_{1} e^{\eta t}$, noting that $b_{2}(\lambda+\kappa)<0$, we get

$$
\begin{aligned}
\mathbb{E}\left|X_{t}\right|^{p} & \leq \frac{e^{-\eta t}}{b_{1}} \mathbb{E} V\left(X_{0}, 0, r_{0}\right)+\frac{e^{-\eta t}}{b_{1}} \mathbb{E} \int_{0}^{t} e^{\lambda_{s}} b_{2}(\lambda+\kappa)\left|X_{t}\right|^{p} \mathrm{~d} s \\
& \leq \frac{e^{-\eta t}}{b_{1}} \mathbb{E} V\left(X_{0}, 0, r_{0}\right) .
\end{aligned}
$$

Therefore, we obtain the required assertion

$$
\limsup _{t \rightarrow \infty} \frac{1}{t} \log \left(\mathbb{E}\left|X_{t}\right|^{p}\right)<0 .
$$

The proof is complete. 


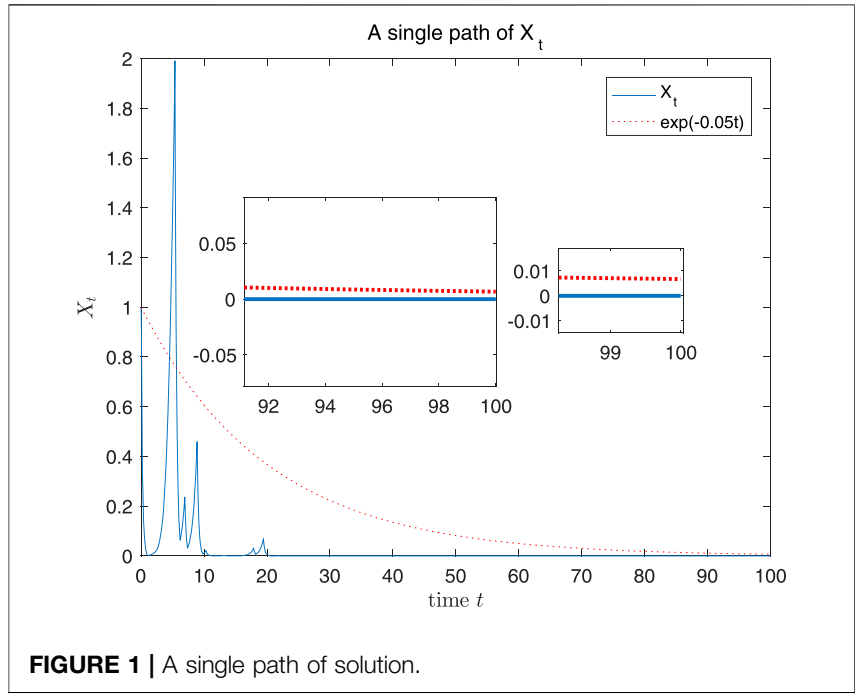

\section{EXAMPLE}

In this section we give a numerical example to illustrate our results.

Example 1. Let $\left\{r_{t}\right\}_{t \geq 0}$ be a right-continuous Markov chain taking values in $\mathbb{S}=\{1,2\}$ with invariant probability measure $\mu_{1}=\mu_{2}=$ $\frac{1}{2}$.

Consider a risky asset, with the price dynamics:

$$
\left\{\begin{array}{l}
\mathrm{d} X_{t}=f\left(X_{t}, t, r_{r}\right) \mathrm{d} t+\sigma\left(t, r_{t}\right) X_{t} \mathrm{~d} B_{t}^{H}, \\
X_{0}=1,
\end{array}\right.
$$

on $t \geq 0$. Here we take $H=0.7$ and

$$
\left\{\begin{array}{l}
f(x, t, i)=-4 x, \sigma(t, i)=\frac{1}{t+1}, \text { if } i=1, \\
f(x, t, i)=[2-\sin (x)] x, \sigma(t, i)=e^{-t}, \text { if } i=2 .
\end{array}\right.
$$

Note that for all $i \in \mathbb{S}, \mathrm{d} X_{t}=f\left(X_{t}, t, i\right) \mathrm{d} t+\sigma(t, i) X_{t} \mathrm{~d} B_{t}^{H}$ satisfy the hypothesises (i)-(v). Then, by Lemma 3.1, it is easy to show that Eq. 29 has a unique solution $\left\{X_{t}\right\}_{t \geq 0}$ as well. Set $V(x$, $t, i)=x^{2}$, for $i=1,2$.

Noting that for some $t_{0}>0$ sufficiently large and all $t>t_{0}$, we have

$$
\begin{aligned}
\mathcal{L}^{(1)} V(x, t, 1) & =V_{x}(x, t, 1) f\left(X_{t}, t, 1\right)+V_{x x}(x, t, 1) \frac{1}{t+1} x D_{s}^{\phi} x \\
& \leq-8 x^{2}+2 \frac{1}{t+1} x\left[x H t^{2 H-1}\right] \\
& =-8 x^{2}+o(1) x^{2}:=\beta_{1} x^{2}
\end{aligned}
$$

and

$$
\begin{aligned}
\mathcal{L}^{(2)} V(x, t, 2) & =V_{x}(x, t, 2) f\left(X_{t}, t, 2\right)+V_{x x}(x, t, 2) e^{-t} x D_{s}^{\phi} x \\
& =2 x^{2}[2-\sin (x)]+o(1) x^{2} \\
& \leq 6 x^{2}+o(1) x^{2}:=\beta_{2} x^{2}
\end{aligned}
$$

Compute

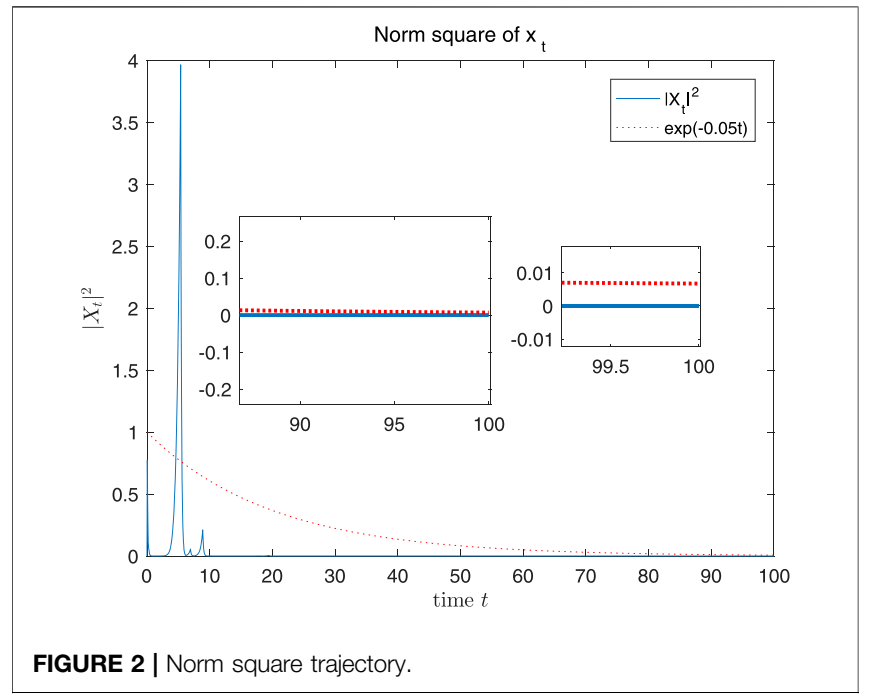

$$
\sum_{i \in \mathbb{S}} \mu_{i} \beta_{i}=\frac{1}{2}(-8+6)+o(1)<0 .
$$

By Theorem 5.2, it's clear that the solution of Eq. 29 is second moment exponential stable. Figures 1, 2 show a single path of the solution and the solution's norm square, respectively.

\section{DATA AVAILABILITY STATEMENT}

The original contributions presented in the study are included in the article/Supplementary Material, further inquiries can be directed to the corresponding author.

\section{AUTHOR CONTRIBUTIONS}

WP contributed to conception and design of the study. WP wrote the first draft of the manuscript. ZZ and WP wrote sections of the manuscript. All authors contributed to manuscript revision, read, and approved the submitted version.

\section{FUNDING}

The research of WP was supported by the Characteristic and Preponderant Discipline of Key Construction Universities in Zhejiang Province (Zhejiang Gongshang University-Statistics).

\section{ACKNOWLEDGMENTS}

The authors are grateful to thank the reviewers for careful reading of the paper and for helpful comments that led to improvement of the first version of this paper. 


\section{REFERENCES}

1. Yin GG, and Zhu C. Hybrid switching diffusions: Properties and applications, Stoch. Model. Appl. Probab. New York: Springer (2010).

2. Mao XR, and Yuan CG. Stochastic differential equations with Markovian switching. South Kensington: Imperial College Process (2006).

3. Hamilton JD. A new approach to the economic analysis of nonstationary time series and the business cycle. Econometrica (1989) 57:357-84. doi:10.2307/ 1912559

4. Mao X. Stability of stochastic differential equations with Markovian switching. Stochastic Process their Appl (1999) 79:45-67. doi:10.1016/s0304-4149(98) 00070-2

5. Yuan C, and Mao X. Stability of stochastic delay hybrid systems with jumps. Eur J Control (2010) 16:595-608. doi:10.3166/ejc.16.595-608

6. Zhou W, Yang J, Yang X, Dai A, Liu H, and Fang JA. pth Moment exponential stability of stochastic delayed hybrid systems with Lévy noise. Appl Math Model (2015) 39:5650-8. doi:10.1016/j.apm.2015.01.025

7. Yuan C, and Mao X. Asymptotic stability in distribution of stochastic differential equations with Markovian switching. Stochastic Process their Appl (2003) 103:277-91. doi:10.1016/s0304-4149(02)00230-2

8. Li X, and Mao X. A note on almost sure asymptotic stability of neutral stochastic delay differential equations with Markovian switching. Automatica (2012) 48:2329-34. doi:10.1016/j.automatica.2012.06.045

9. $\mathrm{Wu} \mathrm{H}$, and Sun J. p-Moment stability of stochastic differential equations with impulsive jump and Markovian switching. Automatica (2006) 42:1753-9. doi:10.1016/j.automatica.2006.05.009

10. Hurst HE. Long-term storage capacity in reservoirs. Trans Amer Soc Civil Eng (1951) 116:400-10. doi:10.1061/taceat.0006518

11. Hurst HE. Methods of using long-term storage in reservoirs. Proc Inst Civil Eng (1956) 5:519-43. doi:10.1680/iicep.1956.11503

12. Mandelbrot BB. The Fractal Geometry of Nature. San Francisco, CA: Freeman (1983).

13. Mandelbrot BB. Fractals and Scaling in Finance: Discontinuity, Concentration, Risk. Berlin: Springer-Verlag (1997).

14. $\mathrm{Hu} \mathrm{Y}$, and $\varnothing \mathrm{ksendal} \mathrm{B.} \mathrm{Fractional} \mathrm{white} \mathrm{noise} \mathrm{calculus} \mathrm{and} \mathrm{applications} \mathrm{to}$ finance. Infin Dimens Anal Quan Probab. Relat. Top. (2003) 06:1-32. doi:10.1142/s0219025703001110

15. Brody DC, Syroka J, and Zervos M. Dynamical pricing of weather derivatives. Quantitative Finance (2002) 2:189-98. doi:10.1088/1469-7688/2/3/302

16. Biagini F, Hu YZ, Øksendal B, and Zhang TS. Stochastic calculus for fractional Brownian motion and applications. London: Springer-Verlag (2008).

17. Mishura YS. Stochastic calculus for Fractional Brownian Motion and related process. Berlin: Springer-Verlag (2008).

18. Li M. Modified multifractional Gaussian noise and its application. Phys Scr (2021) 96:125002. doi:10.1088/1402-4896/aclcf6

19. Li M. Generalized fractional Gaussian noise and its application to traffic modeling. Physica A (2021) 579:1236137. doi:10.1016/j.physa.2021.126138
20. Li M. Multi-fractional generalized Cauchy process and its application to teletraffic. Physica A: Stat Mech its Appl (2020) 550:123982. doi:10.1016/ j.physa.2019.123982

21. Li M. Fractal time series a tutorial review. Math Probl Eng (2010) 2010:157264. doi:10.1155/2010/157264

22. Ghosh MK, Arapostathis A, and Marcus SI. Ergodic control of switching diffusions. SIAM J Control Optim (1997) 35:1952-88. doi:10.1137/ s0363012996299302

23. Skorohod AV. Asymptotic Methods in the Theory of Stochastic Differential Equations. US: American Mathematical Society (1989).

24. Anderson WJ. Continuous-time Markov chain. New York: Springer (1991).

25. Bardet JB, Gurin H, and Malrieu F. Long time behavior of diffusions with Markov switching. ALEA Lat Am J Probab Math Stat (2010) 7:151-70.

26. Cloez B, and Hairer M. Exponential ergodicity for Markov processes with random switching. Bernoulli (2015) 21:505-36. doi:10.3150/13-bej577

27. Alos E, Mazet O, and Nualart D. Stochastic calculus with respect to Gaussian processes. Ann Probab (1999) 29:766-801.

28. Nualart D, and Răşcanu A. Differential equations driven by fractional Brownian motion. Collect Math (2000) 53:55-81.

29. Holdeb H, Øksendal B, Ubøe J, and Zhang T. Stochastic partial differential equations. Boston: Birkhäuser (1996).

30. Duncan TE, Hu Y, and Pasik-Duncan B. Stochastic Calculus for Fractional Brownian Motion I. Theory. SIAM J Control Optim (2000) 38:582-612. doi:10.1137/s036301299834171x

31. Yan L, Pei WY, Pei W, and Zhang Z. Exponential stability of SDEs driven by fBm with Markovian switching. Discrete Cont Dyn-a (2019) 39:6467-83. doi:10.3934/dcds.2019280

32. Mao XR. Stochastic differential equations and applications. New York: Horwood (1997).

33. Arcones MA. On the law of the iterated logarithm for gaussian processes. J Theor Probab (1995) 8:877-903. doi:10.1007/bf02410116

Conflict of Interest: The authors declare that the research was conducted in the absence of any commercial or financial relationships that could be construed as a potential conflict of interest.

Publisher's Note: All claims expressed in this article are solely those of the authors and do not necessarily represent those of their affiliated organizations, or those of the publisher, the editors and the reviewers. Any product that may be evaluated in this article, or claim that may be made by its manufacturer, is not guaranteed or endorsed by the publisher.

Copyright (c) 2021 Pei and Zhang. This is an open-access article distributed under the terms of the Creative Commons Attribution License (CC BY). The use, distribution or reproduction in other forums is permitted, provided the original author(s) and the copyright owner(s) are credited and that the original publication in this journal is cited, in accordance with accepted academic practice. No use, distribution or reproduction is permitted which does not comply with these terms. 